\title{
Grey Wolf Optimizer Based Integer Order Power System Stabilizer for the Enhancement of Transient Stability of 10 machine 39 bus system
}

\author{
P Dhana Selvi ${ }^{1}$, S Suresh Reddy ${ }^{2}$, R Kiranmayi ${ }^{3}$ \\ ${ }^{1}$ Research Scholar, Department of EEE, JNTUA Ananthapuramu, India, Email:dhanaselvi.nbkrist@gmail.com \\ ${ }^{2}$ Professor, Department of EEE, N B K R I S T, India, Email:sanna_suresh@ rediffmail.com \\ ${ }^{3}$ Professor, Department of EEE, JNTUA Ananthapuramu, India, Email:kiranmayi0109@ gmail.com
}

\begin{abstract}
This paper proposes Grey wolf optimizer-based integer order power system stabilizer (GWOIOPSS) for the improvement of transient stability of a multi-machine power system. The gain and the time constants of this controller are tuned using grey wolf optimization algorithm. The proposed controller is applied on 10 machine 39 bus system with fault as a disturbance and implemented in MATLAB/Simulink environment. Speed deviations, electrical power deviations and rotor angle deviations are considered as performance parameters to show the effectiveness of the proposed controller. Performance of this controller is compared with conventional power system stabilizer; the results demonstrated that GWOIOPSS is effectively damping the power system oscillations compared with conventional power system stabilizer.
\end{abstract}

Key words : Grey wolf optimizer algorithm (GWO), Integer order power system stabilizer (IOPSS), conventional power system stabilizer (CPSS).

\section{INTRODUCTION}

In general the power system is highly nonlinear and inter-connected system. Due to interconnections between various areas of power system, the frequency oscillations of one are affecting the frequency of other areas. Depending on magnitude of frequency oscillations, there two stability analysis first one is dynamic stability analysis and second one is transient stability analysis. Transient stability analysis is very important; if this is not improved system collapse will take place. Therefore, transient stability is considered in this paper [1]. Demello et al., proposed excitation control method for stability enhancement [2]. Larsen and Swann proposed power system stabilizer for stability enhancement [3]. These authors proposed different methods for designing of PSS, but after design these parameters of controllers are fixed that means at the time of design only one operating point is considered. Therefore, if operating point of power system changes, the effectiveness of PSS is changing.

$\mathrm{Yu}$ proposed mathematical model for calculation of different operating conditions based on real power, reactive power and terminal voltages [4]. Cheng et al., proposed self tuning power system stabilizer (STPSS) for stability enhancement of multi machine power system [5]. Hsu et al., proposed fuzzy logic controller type power system stabilizer for multi machine power system stability enhancement [6]. In this paper conventional power system stabilizer is replaced with fuzzy logic controller and design of this controller depends upon expert knowledge. Zhang et al., proposed artificial neural network-based PSS for enhancing the stability of power system [7-8]. In this paper tuning of neural network requires past history. These methods require expert knowledge or past data and these are affecting the performance of PSS.

Goldberge proposes application of genetic algorithms for optimal tuning of parameters [9]. Abdel et al., proposed a new method for stability enhancement of power system [10]. In this method the parameters of power system stabilizer are designed and implemented using genetic algorithm optimization technique. Abido et al., also proposed genetic algorithm-based power system stabilizer for stability enhancement [11]. Abido et al., proposed hybrid power system stabilizer using genetic algorithm for oscillations damping [12]. Afzalian et al., proposed neuro fuzzy power system stabilizer using genetic algorithm for stability enhancement [13]. Lakshmi et al., proposed fuzzy logic and genetic algorithm-based power system stabilizer for multi machine stability [14]. Wang proposed modified genetic algorithm based PID controller for oscillation damping [15]. Andreiou at al., proposed lyapunov and genetic algorithm based power system stabilizer for stability enhancement [16]. Dhana selvi et al., proposed Grey wolf optimizer algorithm based PSS for multi machine system stability enhancement [17-20]. All these papers propose different methods for 
P Dhana Selvi et al., International Journal of Emerging Trends in Engineering Research, 8(9), September 2020, 6415 - 6422

stability enhancement, but are facing problem due to changes in the operating conditions or changes in the fault locations and types.

In this paper, GWOIOPSS is proposed for the enhancement of transient stability under fault conditions and performance is compared with CPSS through performance indices.

This paper is organized into four sections: section 1 is the introduction; section 2 is over view of IOPSS \& implementation of GWO on IOPSS, section 3 shows simulation diagram \& results with discussion and section 4 is the conclusion.

\section{OVER VIEW OF IOPSS \& GWO IMPLEMENTATION}

Integer order power system stabilizer is combination of three parts. First part is the gain block which provides suitable supportive damping power the respective generating station to damp the oscillations. Second part is the lead-lag compensator which provides suitable leading angle to the gain, so that the gain will appear at right time and provide aid for generating station. Third part is washout block which acts as a electronic switch, this block avoids mal-operation of IOPSS that means if no oscillations no damping is required, this block decides damping required or not and based that decision it will close or open the IOPSS.

\subsection{Implementation of GWO algorithm for IOPSS}

The effectiveness of the proposed controller depends on the parameters of gain and washout block time constants. To get optimized values for this controller, GWO algorithm [21] is applied. In the optimization process, fitness function is considered as a reciprocal of integral time area error. Implementation of GWO is as follows:

1. Initialization of requiring parameters for Iterations, searching agents, tuning parameters, ranges for $\alpha, \beta, \Delta \& \omega$, integral time area error.

2. Run the simulation file over a specified period with initial values and re-evaluate ITAE.

3. $\alpha, \beta \& \Delta$ Values are updated.

4. Update the searching positions of $\alpha, \beta, \Delta \& \omega$ using the following equations:

$$
\begin{aligned}
& A_{1}=2 a_{1} r_{n 1}-a_{1} \\
& B_{1}=2 r_{n 2} \\
& C_{\alpha}=B_{1} P_{\alpha}-\text { Best }_{\alpha} \\
& C_{\beta}=B_{1} P_{\beta}-\text { Best }_{\alpha} \\
& C_{\delta}=B_{1} P_{\delta}-\text { Best }_{\alpha} \\
& B_{\omega}=B_{1} P_{\omega}-\text { Best }_{\alpha}
\end{aligned}
$$

5. Update the parameters of PSS with new values

6. Run simulation file with updated PSS parameters and evaluate ITAE and repeat the procedure from step 3.
7. Stop the optimization procedure once iterations are completed.

The parameters of power system stabilizer gains and time constants of these controllers are optimized using the GWO algorithm as per the given procedure. Here integral time area error is the measure of speed deviations.

\section{Simulation diagRAM \& RESUlts}

To show the effectiveness of the proposed controller, fault is created in line between buses $35 \& 22$ at time 3 to 3.01 seconds. The simulation results are shown in figures 2 to 29 .

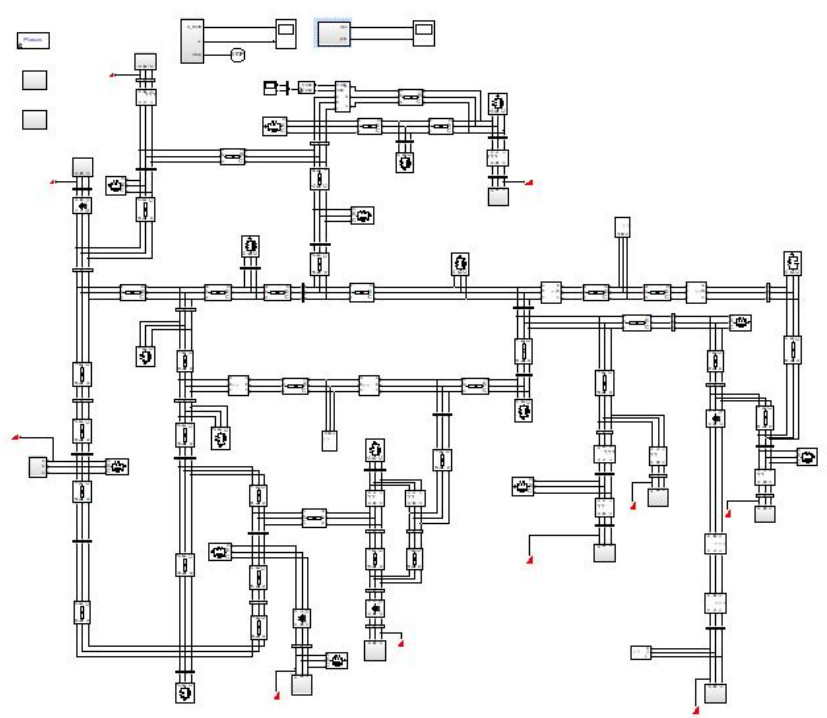

Figure 1: MATLAB/Simulink diagram of 10 machine 39 bus system.

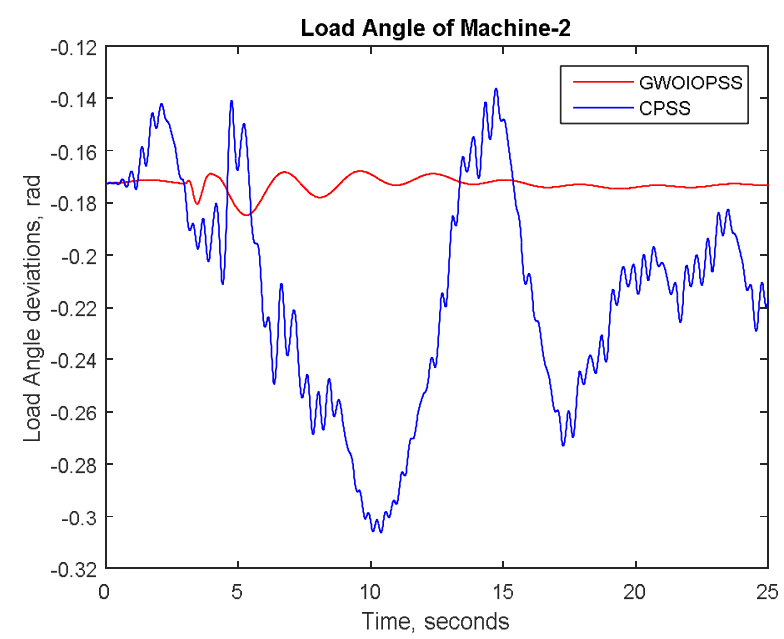

Figure 2: Load angle deviations of machine 2 with respect to machine 1 


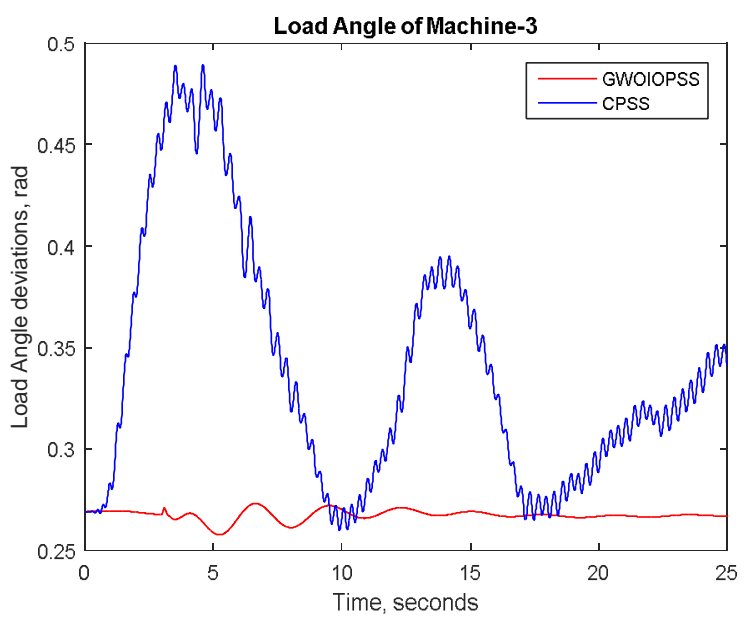

Figure 3: Load angle deviations of machine 3 with respect to machine 1

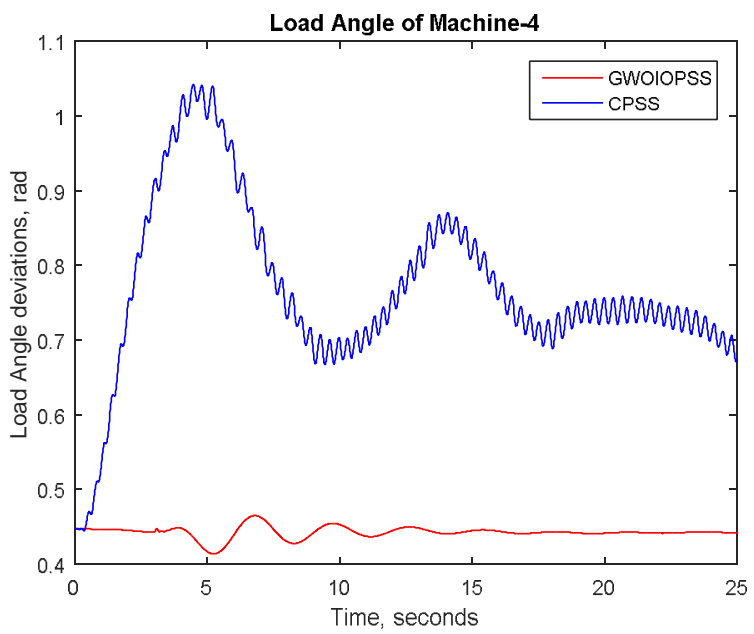

Figure 4: Load angle deviations of machine 4 with respect to machine 1

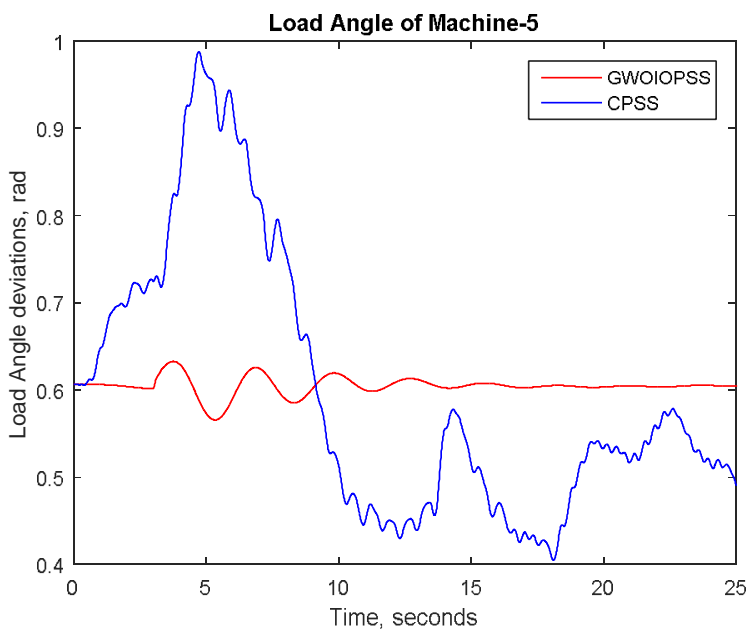

Figure 5: Load angle deviations of machine 5 with respect to machine 1

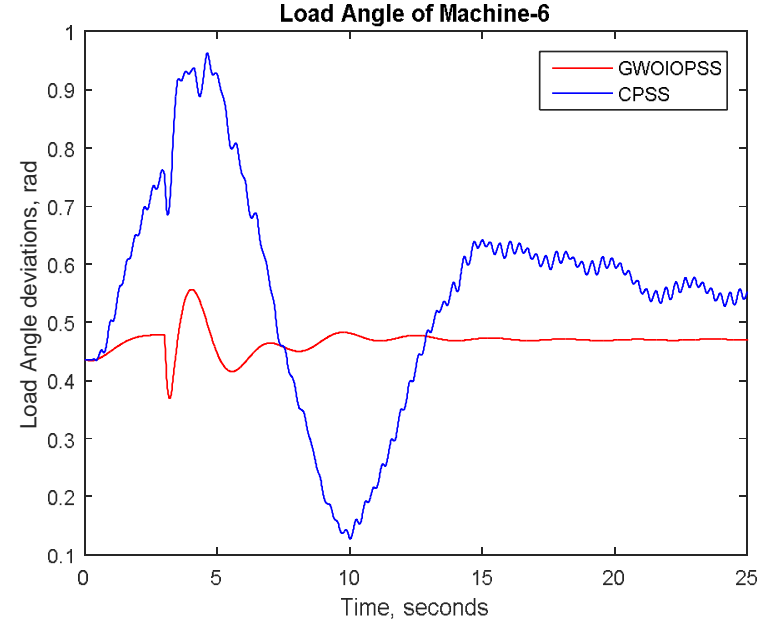

Figure 6: Load angle deviations of machine 6 with respect to machine 1

Figure 2 shows the load angle deviations of machine 2 with respect to machine1, from this the proposed controller effectively reducing the oscillation of loan angle as compared with conventional PSS (CPSS).

Figure 3 shows the load angle deviations of machine 3 with respect to machine1, from this the proposed controller effectively reducing the oscillation of loan angle as compared with CPSS.

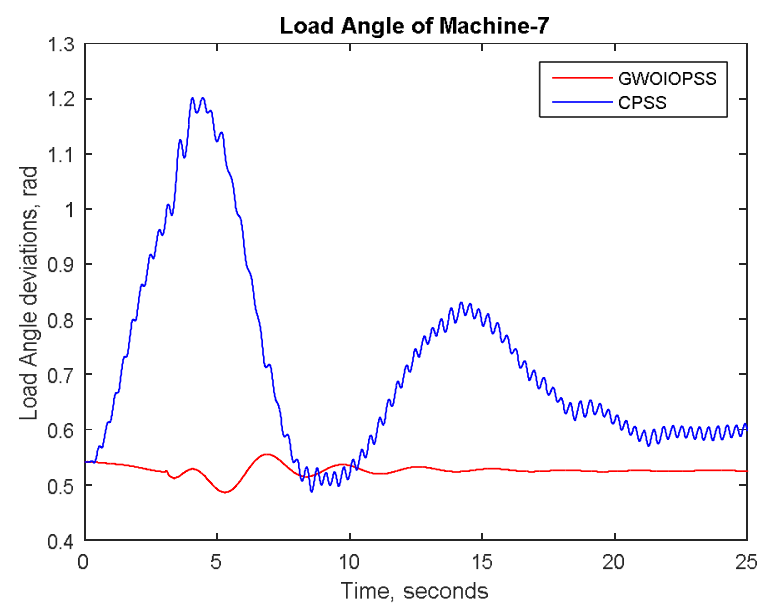

Figure 7: Load angle deviations of machine 7 with respect to machine 1

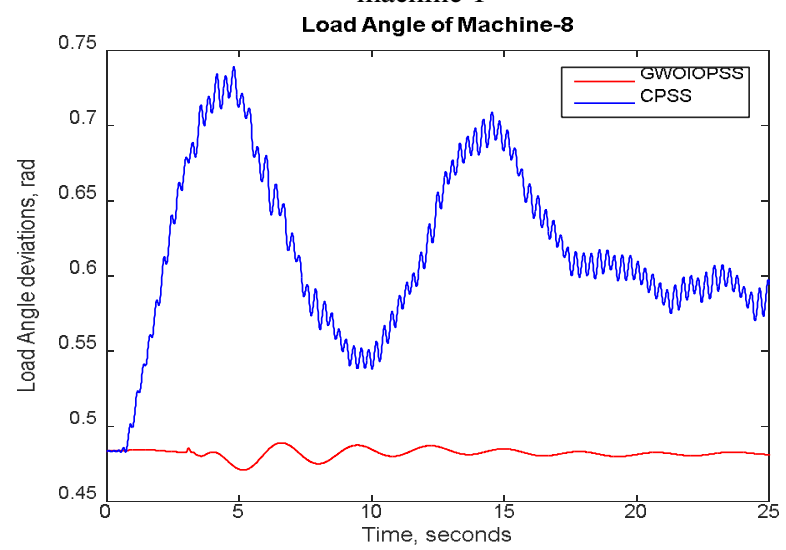

Figure 8: Load angle deviations of machine 8 with respect to machine 1 


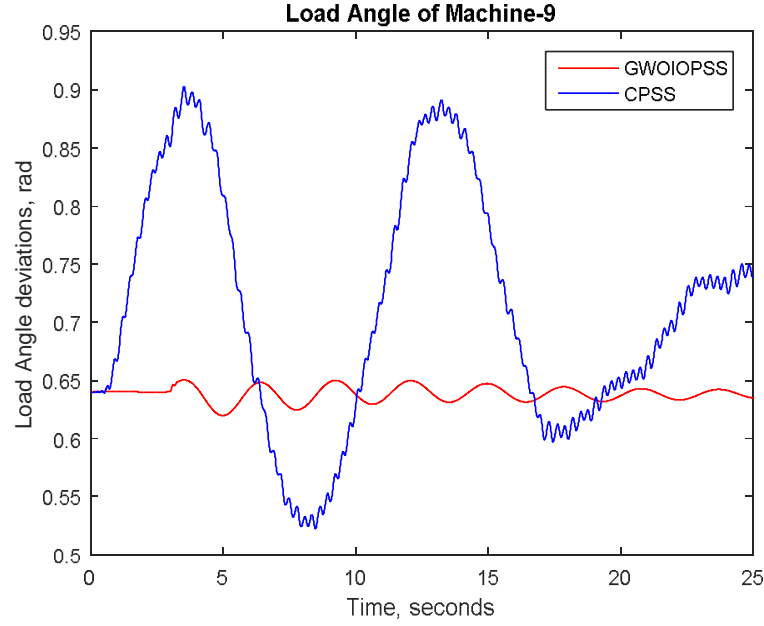

Figure 9: Load angle deviations of machine 9 with respect to machine 1

Figure 4 shows the load angle deviations of machine 4 with respect to machine1, from this the proposed controller effectively reducing the

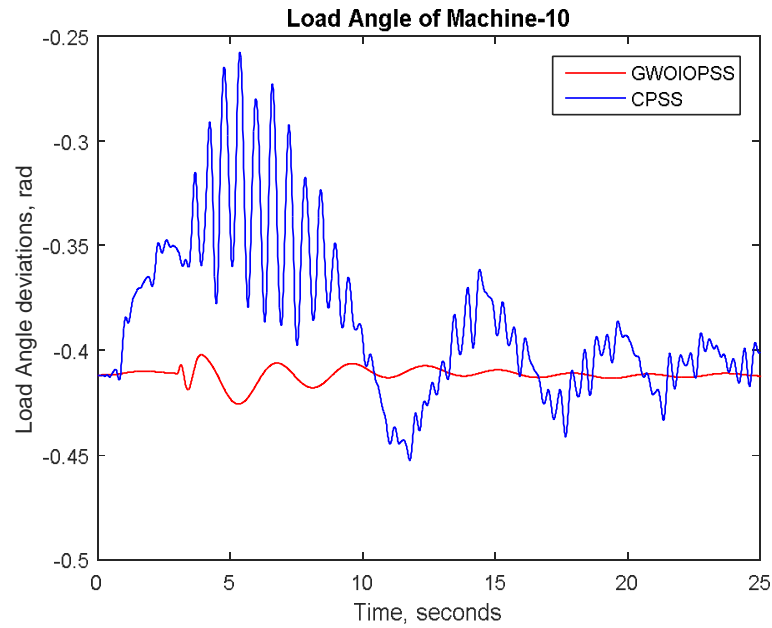

Figure 10: Load angle deviations of machine 10 with respect to machine 1

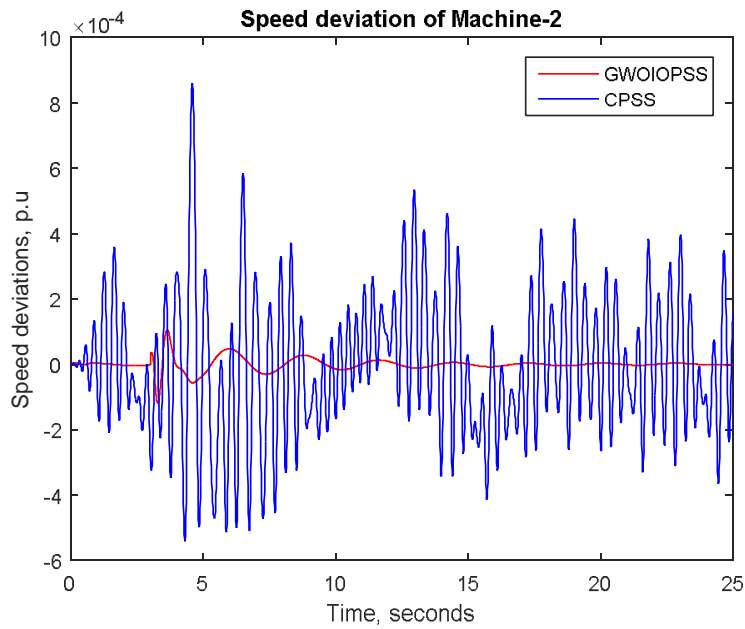

Figure 11: Rotor speed deviations of machine 2 with respect to machine 1

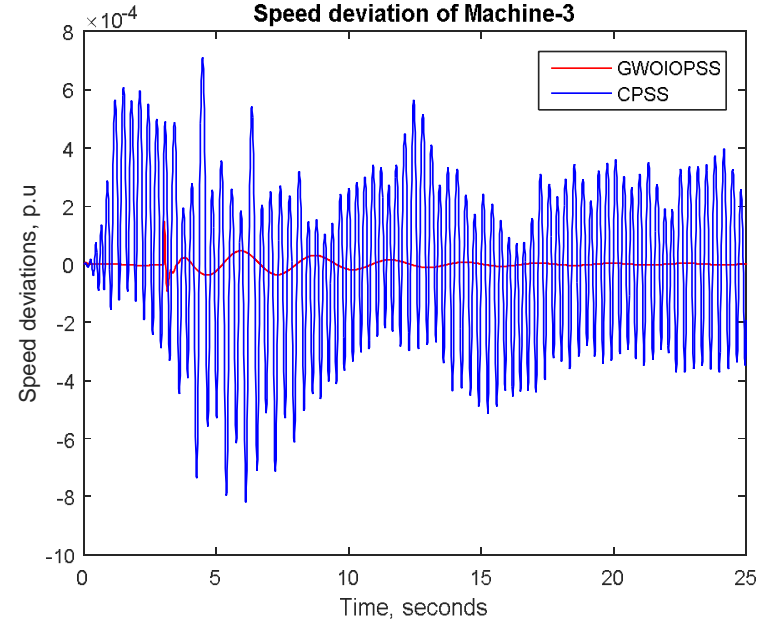

Figure 12: Rotor speed deviations of machine 3 with respect to machine 1

oscillation of loan angle as compared with conventional PSS (CPSS).

Figure 5 shows the load angle deviations of machine 5 with respect to machine1, from this the proposed controller effectively reducing the oscillation of loan angle as compared with CPSS.

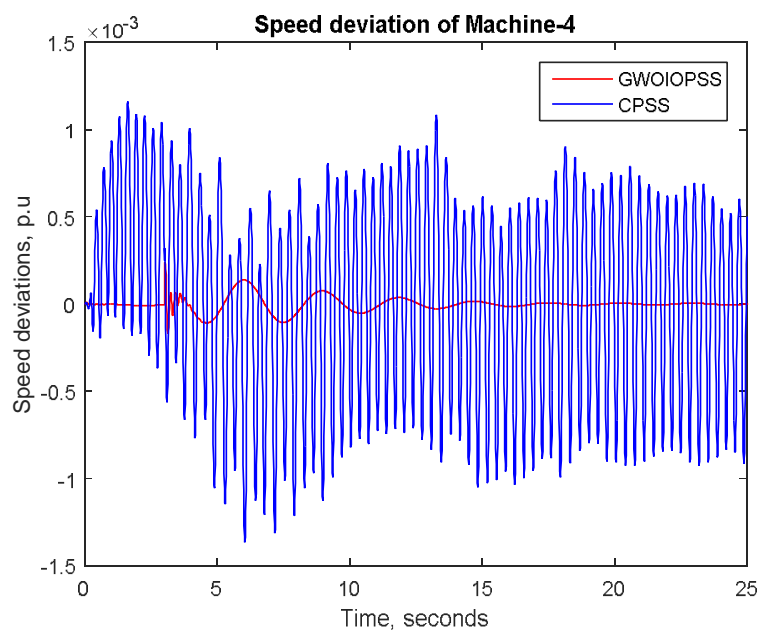

Figure 13: Rotor speed deviations of machine 4 with respect to machine 1

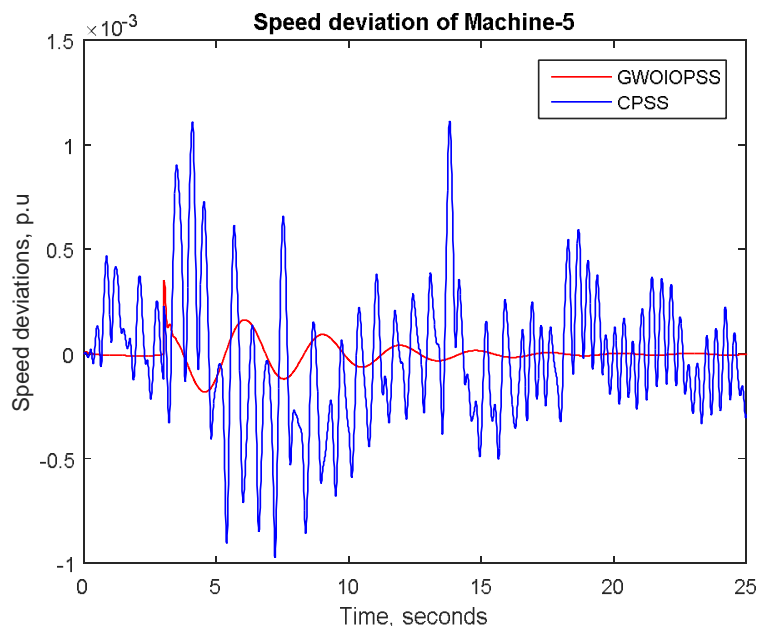

Figure 14: Rotor speed deviations of machine 5 with respect to machine 1 


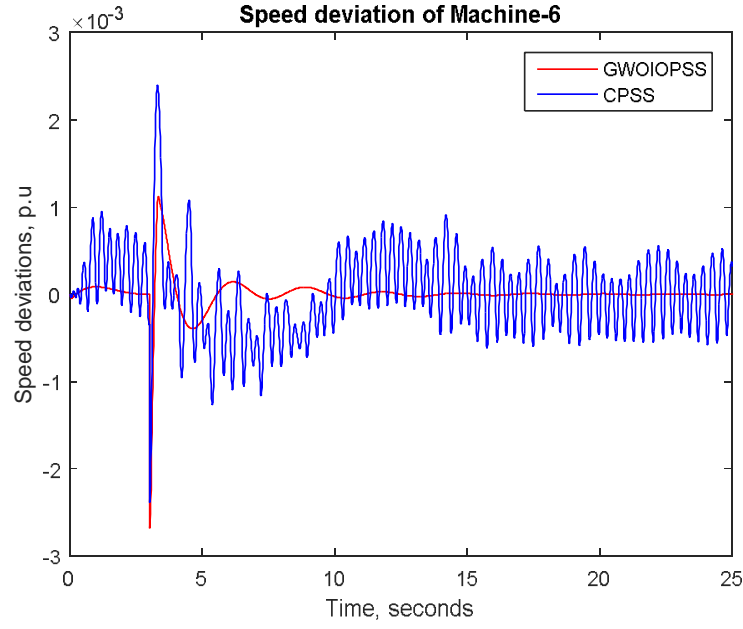

Figure 15: Rotor speed deviations of machine 6 with respect to machine 1

Figure 6 shows the load angle deviations of machine 6 with respect to machine 1 , from this the proposed controller effectively reducing the oscillation of loan angle as compared with conventional PSS (CPSS).

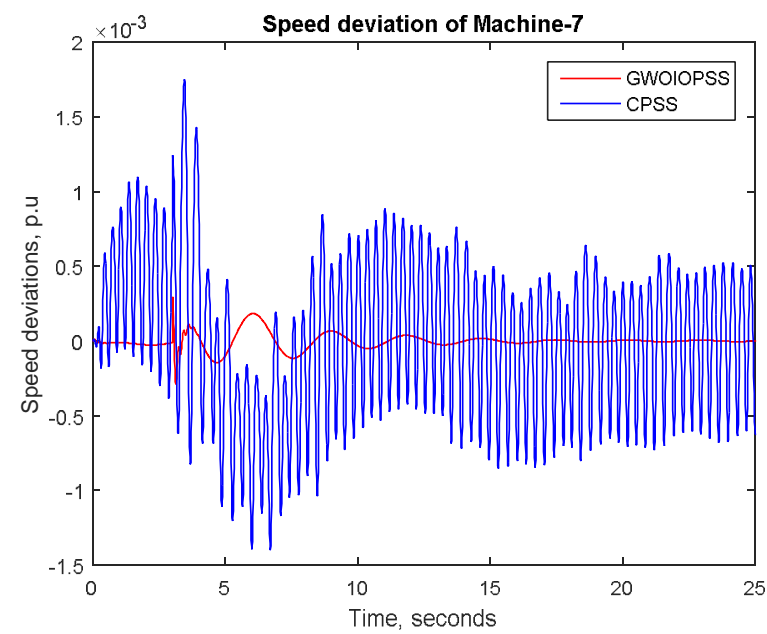

Figure 16: Rotor speed deviations of machine 7 with respect to machine 1

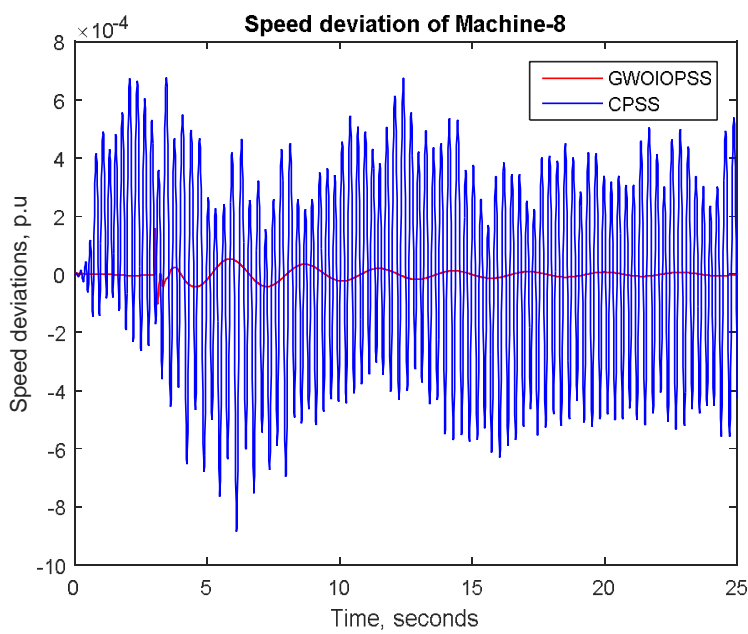

Figure 17: Rotor speed deviations of machine 8 with respect to machine 1
Figure 7 shows the load angle deviations of machine 7 with respect to machine1, from this the proposed controller effectively reducing the oscillation of loan angle as compared with CPSS.

Figure 8 shows the load angle deviations of machine 8 with respect to machine1, from this the proposed controller effectively reducing the oscillation of loan angle as compared with conventional PSS (CPSS).

Figure 9 shows the load angle deviations of machine 9 with respect to machine1, from this the proposed controller effectively reducing the oscillation of loan angle as compared with CPSS.

Figure 10 shows the load angle deviations of machine 10 with respect to machine1, from this the proposed controller effectively reducing the oscillation of loan angle as compared with conventional PSS (CPSS). Figure 11 shows the speed deviations of machine 2 with respect to machine1, from this the proposed controller effectively reducing the oscillation of speed as compared with CPSS. Figure 12 shows the speed deviations of machine 3 with respect to machine1, from this the proposed controller effectively reducing the oscillation of speed as compared with CPSS. Figure 13 shows the speed deviations of machine 4 with respect to machine 1 , from this the proposed controller effectively reducing the oscillation of speed as compared with CPSS.

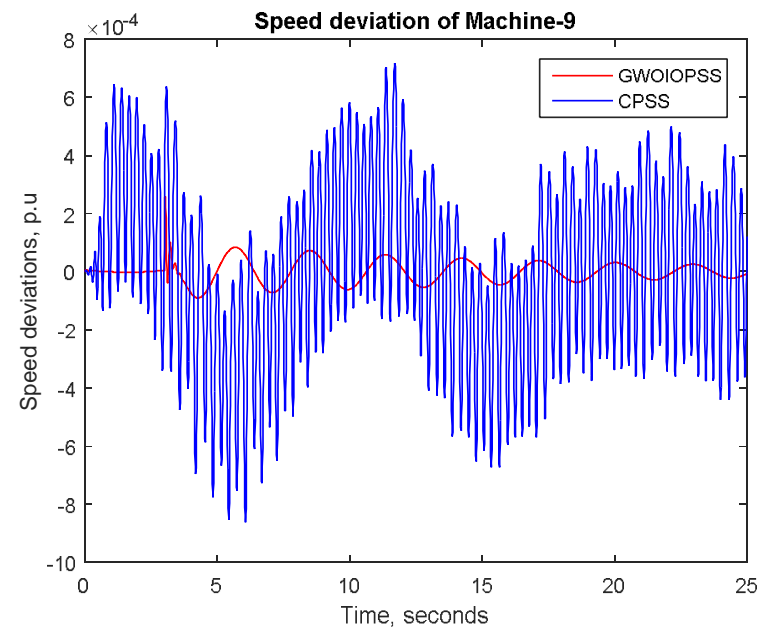

Figure 18: Rotor speed deviations of machine 9 with respect to machine 1

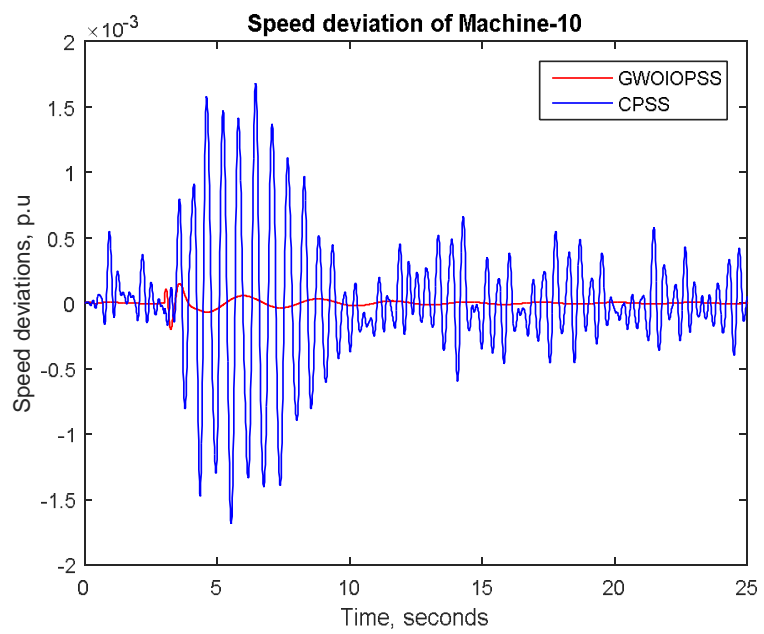

Figure 19: Rotor speed deviations of machine 10 with respect to machine 1 


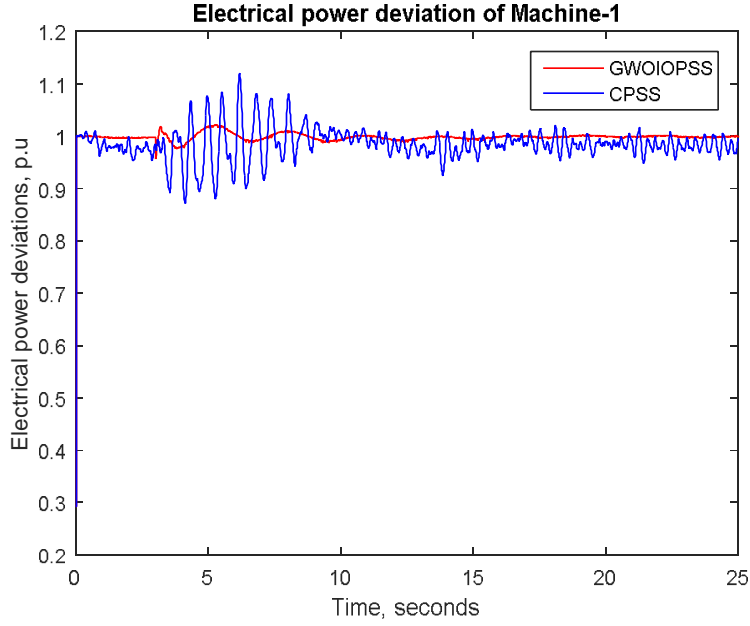

Figure 20: Electrical power deviations of machine 1 with respect to Time

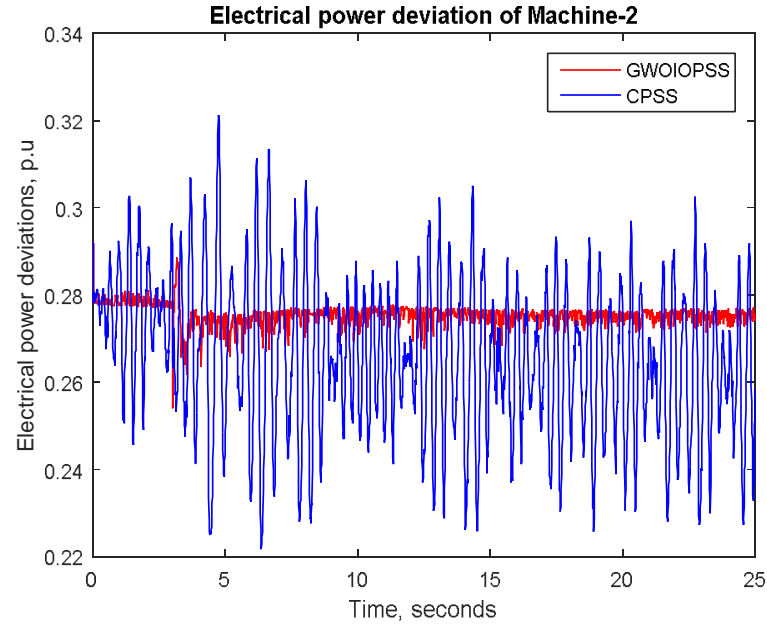

Figure 21: Electrical power deviations of machine 2 with respect to Time

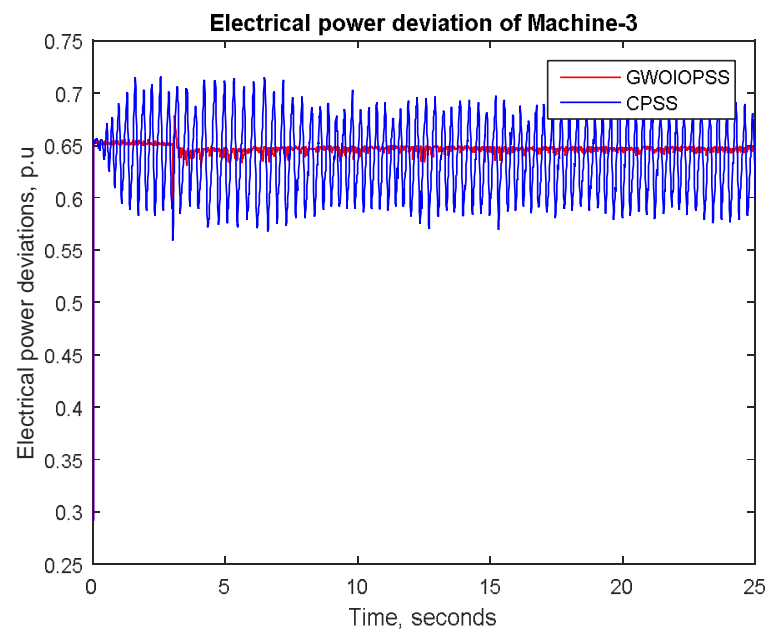

Figure 22: Electrical power deviations of machine 3 with respect to Time

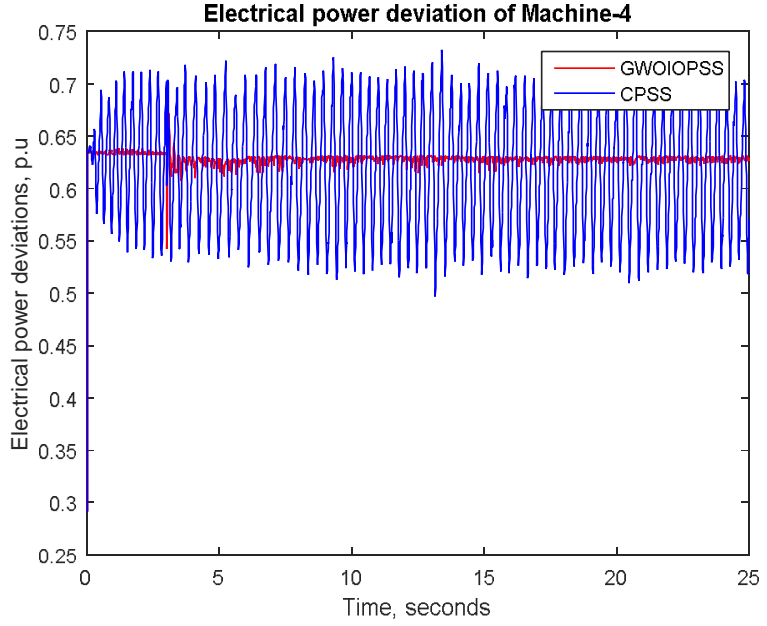

Figure 23: Electrical power deviations of machine 4 with respect to Time

Figure 14 shows the speed deviations of machine 5 with respect to machine1, from this the proposed controller effectively reducing the oscillation of speed as compared with CPSS. Figure 15 shows the speed deviations of machine 6 with respect to machine 1 , from this the proposed controller effectively reducing the oscillation of speed as compared with CPSS.

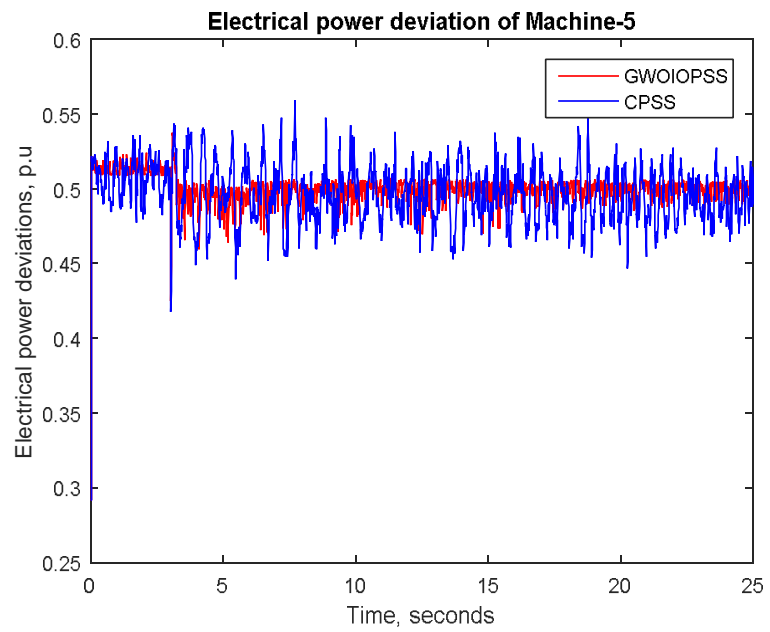

Figure 24: Electrical power deviations of machine 5 with respect to Time

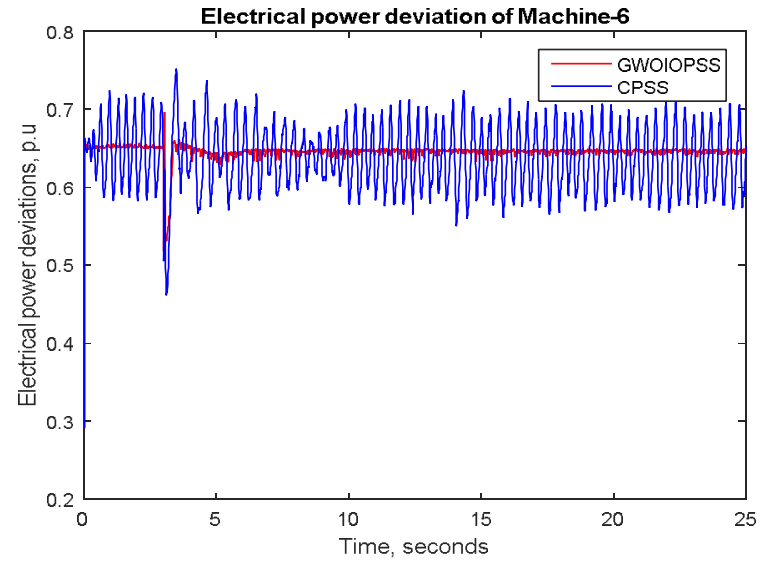

Figure 25: Electrical power deviations of machine 6 with respect to Time 


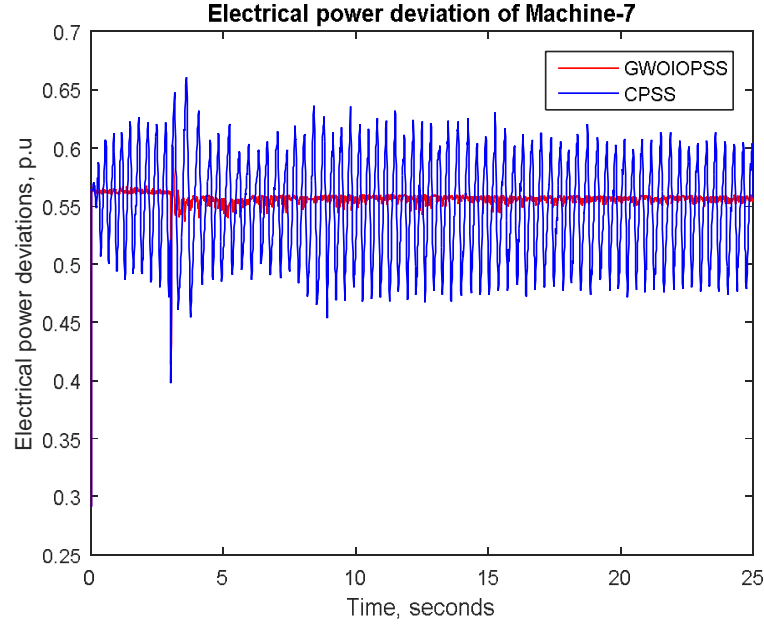

Figure 26: Electrical power deviations of machine 7 with respect to Time

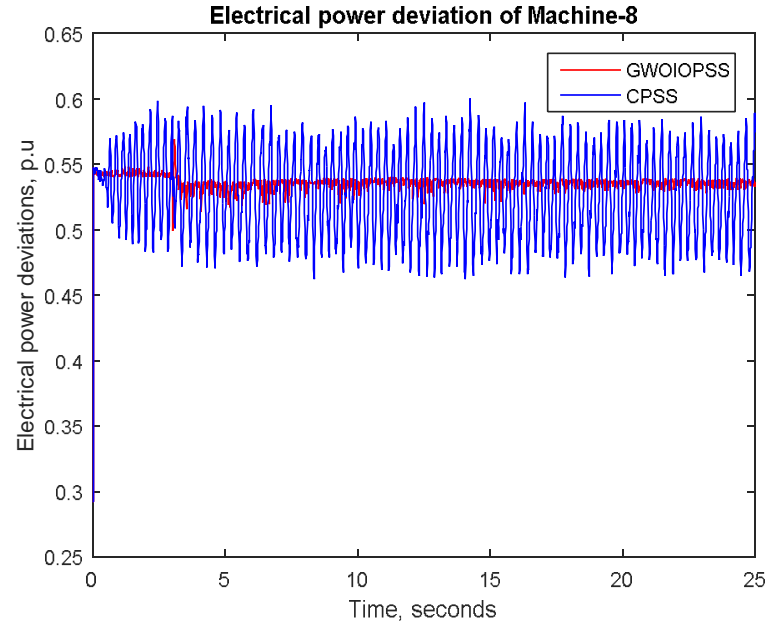

Figure 27: Electrical power deviations of machine 8 with respect to Time

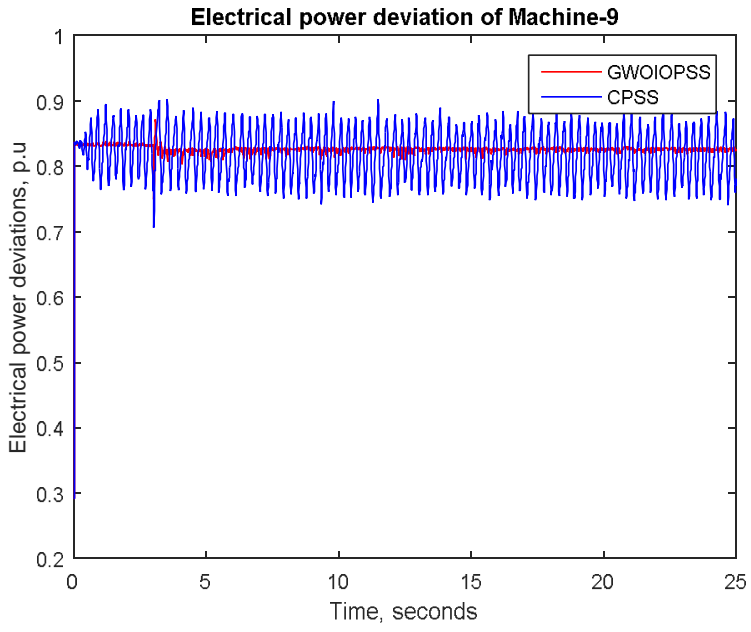

Figure 28: Electrical power deviations of machine 9 with respect to Time

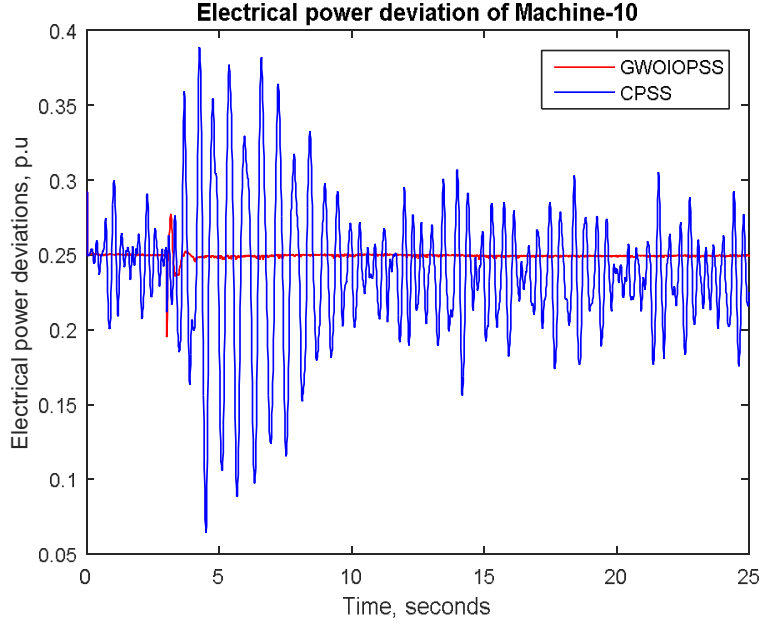

Figure 29: Electrical power deviations of machine 10 with respect to Time

Figure 16 shows the speed deviations of machine 7 with respect to machine1, from this the proposed controller effectively reducing the oscillation of speed as compared with CPSS. Figure 17 shows the speed deviations of machine 8 with respect to machine1, from this the proposed controller effectively reducing the oscillation of speed as compared with CPSS.

Figure 18 shows the speed deviations of machine 9 with respect to machine1, from this the proposed controller effectively reducing the oscillation of speed as compared with CPSS. Figure 19 shows the speed deviations of machine 10 with respect to machine 1 , from this the proposed controller effectively reducing the oscillation of speed as compared with CPSS.

Figure 20 shows the electrical power deviations of machine 1 with respect to time, from this the proposed controller effectively reducing the oscillation of electrical power as compared with CPSS. Figure 21 shows the electrical power deviations of machine 2 with respect to time, from this the proposed controller effectively reducing the oscillation of electrical power as compared with CPSS. Figure 22 shows the electrical power deviations of machine 3 with respect to time, from this the proposed controller effectively reducing the oscillation of electrical power as compared with CPSS. Figure 22 shows the electrical power deviations of machine 3 with respect to time, from this the proposed controller effectively reducing the oscillation of electrical power as compared with CPSS. Figure 23 shows the electrical power deviations of machine 4 with respect to time, from this the proposed controller effectively reducing the oscillation of electrical power as compared with CPSS. Figure 24 shows the electrical power deviations of machine 5 with respect to time, from this the proposed controller effectively reducing the oscillation of electrical power as compared with CPSS. Figure 25 shows the electrical power deviations of machine 6 with respect to time, from this the proposed controller effectively reducing the oscillation of electrical power as compared with CPSS. Figure 26 shows the electrical power deviations of machine 7 with respect to time, from this the proposed controller effectively reducing the oscillation of electrical power as compared with CPSS. Figure 27 shows the electrical power deviations of machine 8 with respect to time, from this the proposed controller effectively reducing the oscillation of electrical power as compared with CPSS. Figure 28 shows the electrical power deviations of machine 9 with respect to time, from this the proposed controller effectively reducing the oscillation of electrical power as compared with CPSS. Figure 29 shows the electrical power deviations of machine 10 with 
P Dhana Selvi et al., International Journal of Emerging Trends in Engineering Research, 8(9), September 2020, 6415 - 6422

respect to time, from this the proposed controller effectively reducing the oscillation of electrical power as compared with CPSS.

\section{CONCLUSION}

This paper proposed GWOIOPSS controller for 10 machine 39 bus system for the enhancement of transient stability. The proposed controller is implemented in MATLAB/Simulink and the performance is compared with conventional power system stabilizer using three performance parameters. Performance parameters shown that the proposed controller is effectively damping the oscillations of performance parameters as compared with CPSS.

\section{REFERENCES}

1. P. Kundur, "Power System Stability and Control", McGraw Hill Inc. 1993.

2. F.P. Demello and C. Concordia, "Concept of synchronous machinestability as affected by excitation control", IEEE Trans. PAS Vol. 88,1969, pp 316-329.

3. E.V. Larsen and D.A. Swann, “ Applying Power System Stabilizers", Part I- III, IEEE Trans. PAS Vol. 100, no 6, 1981, pp 3017-3046.

4. Y.N. Yu, Electric Power System Dynamics, Academic Press, 1983.

5. Cheng S, Malik O.P., Hope G.S., "Design of self-tuning PID stabilizerfor a multimachine power system ", IEE Proceedings, Part C,1986;133(4),pp 176-185.

6. Hsu Y.Y.,Liou K.L., Hsu Y.Y.,Cheng C.V., , "Design of fuzzy power system stabilizers for multimachine power systems ", IEE Proceedings,Part C,1990, 137(3) .

7. Y. Zhang, G.P. Chen, O.P. Malik and G.S. Hope, “ An artificial neural based adaptive power system stabilizer", IEEE Transactions on Energy Conversion, Vol.8,No.1, March 1993,pp.71-77.

8. Y.Zhang, O.P.Malik and G.P.Chen, " Artificial neural network power system stabilizers in multi-machine power system environment ", IEEE Transactions on Energy Conversion, Vol. 10, No.1, March 1995, pp.147-154.

9. D. E. Goldberge, Genetic Algorithms in Search, Optimization, and

10. Machine Learning, Addison-Wesley publishing Company Inc., January 1989.

11. Y.L.Abdel-Magid, M. Bettayeb, M.M. Dawoud, "Simultaneous stabilization of power systems using genetic algorithm", IEE Proceedings-Generation,Transmission and Distribution, Vol.144, No.1,January 1997.

12. M.A.Abido, Y.L.Abdel-Mazid, "A Genetic- Based Power System Stabilizer", Electric Machines and Power Systems,, 26, 1998, pp 559-571.

13. M.A.Abido and Y.L.Abdel-Magid , “ Hybridizing rule-based power system stabilizers with genetic algorithms", IEEE Transactions on Power Systems, Vol.14, May 1999,pp. 600-607.

14. Afzalian and D.A Linkens, "Training of neuro-fuzzy power system stabilizers using genetic algorithms", Electric Power and Energy Systems 22,2000,pp. 93-102.

15. P.Lakshmi and M. Abdullah Khan, "Stability enhancement of a multi-machine power system using fuzzy logic based power system stabilizer tuned through genetic algorithm ", Electric Power and Energy Systems, 22,2000,pp. 130-145.

16. Y.P.Wang,N. R. Watson and H. H. Chong, “ Modified genetic algorithm to design of an optimal PID controller for AC-DC transmission systems ", International Journal of Electrical Power and Energy Systems, Volume 24, Jan 2002, pp. 59- 69.

17. Andreoiu, K. Bhattacharya, "Robust tuning of power system stabilizers using a Lyapunov method based genetic algorithm", IEE Proceedings on Generation, Transmission and Distribution, Vol.149, No.5, September 2002, pp. 585- 592. Gainesville. Florida, 2007, pp.162-173.

18. P Dhanaselvi, S Suresh Reddy \& R Kiranmayi, "optimal tuning of power system stabilizer parameters using GWO algorithm", Lecture notes in electrical engineering, Vol.569, (2020), pp.347-356.

19. Ramprasad V, k Ravikanth," Wind Power Influence on the Small Signal Stability of Grid Integrated Distribution Systems", International Journal of Emerging Trends in Engineering Research, vol.8, No.7,(2020), pp.3569-3574

20. Z. Kamis, M.R.A. Ghani, M. Sulaiman, M.N. Kamarudin, H.N.M. Shah," PI Controlled SVC for Power System Stability", International Journal of Emerging Trends in Engineering Research, vol.8, No.8,(2020), pp. $4744-4749$

21. S Mirjalili, S M Mirjalili, A Lewis. "Grey Wolf Optimizer". Advances in Engineering Software, vol.69, no.1, (2014), pp.46-61. 